\title{
Incontinences et obésité
}

\author{
G. Amarenco \\ (c) Springer-Verlag France 2010
}

\section{0 à $57 \%$ d'incontinence urinaire. $14,5 \%$ d'obèses. $10 \%$ d'incontinence fécale}

Ces chiffres illustrent bien le vrai problème de santé publique que posent ces affections, problèmes de risques vitaux en ce qui concerne l'obésité et l'incontinence neurologique, problèmes fonctionnels et de qualité de vie dans tous les cas.

C'est dire la nécessité, outre de développer des politiques de prévention, de préciser au mieux les différents déterminants qui sous-tendent ces affections.

Au-delà de certains facteurs génétiques et environnementaux, obésité et incontinences partagent ainsi certains mécanismes physiopathologiques. En effet, les relations entre incontinence et obésité ne se résument pas forcément à un simple facteur " mécaniciste » où l'excès de pression intra-abdominale ou intravésicale induite par la surcharge pondérale dépasse les possibilités résistives des appareils sphinctériens urétraux ou anaux [1,2]. L'association de ces troubles est de fait beaucoup plus complexe et procède de mécanismes de régulation plus intimes.

De nombreuses études épidémiologiques ont mis en évidence la relation entre syndrome métabolique, intriqué avec l'obésité et troubles urinaires tout particulièrement à type d'incontinence [3-7]. Sur le plan physiopathologique, le rôle de l'hyperglycémie sur les neurones parasympathiques des ganglions pelviens, dont on connait l'action sur le détrusor et le sphincter urétral, a été souligné. Une aptoptose accélérée de ces structures induit une perte neuronale déterminant une perte de contrôle de la motricité vésicale, et par voie de conséquence un déséquilibre de la balance sympathique-parasympathique. L'artériosclérose des vaisseaux pelviens conduisant à une ischémie chronique de la vessie et de la prostate, l'existence d'une neuropathie dans le cadre d'un diabète associé responsable d'une atteinte neurogène

\footnotetext{
G. Amarenco $(\bowtie)$

Service de neuro-urologie et d'explorations périnéales, unité de recherche Er6 UPMC,

hôpital Tenon, AP-HP, France

e-mail : gerard.amarenco@rth.aphp.fr
}

sensitive vésicale spécifique, l'hyperinsulinémie avec augmentation des facteurs de croissance insuline-like favorisant la mitose prostatique sont autant de facteurs incriminés.

Mais plus certainement, le déterminant physiopathologique commun entre obésité et troubles fonctionnels urinaires est bien probablement une dysrégulation générale du système nerveux autonome. Ainsi, une « dysautonomie » ne se manifestant que par des troubles sphinctériens a été récemment isolée dans le syndrome clinique d'hyperactivité vésicale [8]. Des anomalies spécifiques des voies végétatives sympathiques et parasympathiques ont été décrites au cours de l'obésité tant sur le plan expérimental que clinique [9-12]. Les études cliniques faisant appel aux tests cardiovasculaires de la fonction autonome (étude de la variabilité R-R, ratio 30/15, Valsalva, Tilt test, cold pressor test...) sont intéressantes dans la confirmation d'une dysrégulation végétative dans différentes populations de patients.

Ainsi, un continuum étiopathogénique entre ces affections (incontinence-obésité-diabète-syndrome métaboliquehypertrophie prostatique) est plausible avec expression clinique différenciée des différents symptômes en fonction du degré d'altération de l'équilibre autonomique et des co-facteurs associés.

Cela ouvre, sans nul doute, la voie à de nombreux travaux tant sur le plan épidémiologique que des explorations cliniques et possiblement dans les années à venir sur le plan thérapeutique.

\section{Références}

1. Rohrmann S, Smit E, Giovannucci E, et al (2004) Associations of obesity with lower urinary tract symptoms and non cancer prostate surgery in the Third National Health and Nutrition Examination Survey. Am J Epidemiol 159:390

2. Erekson EA, Sung VW, Myers DL (2008) Effect of body mass index on the risk of anal incontinence and defecatory dysfunction in women Am J Obstet Gynecol 5:596.e1-4

3. Giovannucci E, Rimm EB, Chute CG, et al (1994) Obesity and benign prostatic hyperplasia. Am J Epidemiol 140:989

4. Hammarsten J, Hogstedt B, Holthuis N, et al (1998) Components of the metabolic syndrome-risk factors for the development of benign prostatic hyperplasia. Prostate Cancer Prostatic Dis 1:157

5. Parsons JK, Carter HB, Partin AW, et al (2006) Metabolic factors associated with benign prostatic hyperplasia. J Clin Endocrinol Metab 91:2562 
6. Rohrmann S, Smit E, Giovannucci E, et al (2004) Associations of obesity with lower urinary tract symptoms and non cancer prostate surgery in the Third National Health and Nutrition Examination Survey. Am J Epidemiol 159:390

7. Kupelian V, McVary KT, Kaplan SA, et al (2009) Association of lower urinary tract symptoms and the metabolic syndrome: results from the Boston Area Community Health Survey. J Urol 182:616-24; discussion 624-5

8. Hubeaux K, Deffieux X, Ismael SS, et al (2007) Autonomic nervous system activity during bladder filling assessed by heart rate variability analysis in women with idiopathic overactive bladder syndrome or stress urinary incontinence. J Urol 6:2483-7
9. Rahmouni K (2010) Obesity, sympathetic overdrive, and hypertension: the leptin connection. Hypertension 4:844-5

10. Thayer JF, Yamamoto SS, Brosschot JF (2009) The relationship of autonomic imbalance, heart rate variability and cardiovascular disease risk factors. Int $\mathbf{J}$ Cardiol [Epub]

11. Yasuhiko Minokoshi, Masayuki Saito, Takashi Shimazu (1986) Metabolic and morphological alterations of brown adipose tissue after sympathetic denervation in rats. J Autonom Nerv Syst 3:197-204

12. Chih Jen Chang, Jin-Shang Wu, Feng-Hwa Lu, et al (1998) Sympathetic hyperactivity in Wistar rats with insulin-resistance. J Autonom Nerv Syst 11:116-9 